\title{
Microwave pretreatment of lignocellulosic material in cholinium ionic liquid for efficient enzymatic saccharification
}

Kazuaki Ninomiya $^{\mathrm{a}}$, Takashi Yamauchi ${ }^{\mathrm{b}}$, Chiaki Ogino ${ }^{\mathrm{c}}$, Nobuaki Shimizu ${ }^{\mathrm{a}}{ }^{*}, \mathrm{Kenji}$ Takahashi $^{\text {b* }}$

anstitute of Nature and Environmental Technology, Kanazawa University, Kakuma-machi, Kanazawa 920-1192, Japan

${ }^{\mathrm{b}}$ Division of Material Engineering, Graduate School of Natural Science and Technology, Kanazawa University, Kakuma-machi, Kanazawa 920-1192, Japan

${ }^{c}$ Department of Chemical Science and Engineering, Graduate School of Engineering, Kobe University, 1-1 Rokkodaicho, Nada-ku, Kobe 657-8501, Japan

*Corresponding authors:

N. Shimizu

Tel.: +81 76234 4807; fax: +81 762344829

E-mail address: nshimizu@t.kanazawa-u.ac.jp

K. Takahashi

Tel.: +81 76234 4828; fax: +8176234 4829

E-mail address: ktkenji@t.kanazawa-u.ac.jp 


\begin{abstract}
We demonstrated that the enzymatic hydrolysis of cellulose after microwave pretreatment of lignocellulosic material in ionic liquids (ILs) is drastically enhanced compared with that after conventional thermal pretreatment in ILs. Three types of cholinium ILs, choline formate (ChFor), choline acetate (ChOAc), and choline propionate (ChPro), were examined. The cellulose saccharification percentage was approximately $20 \%$ for kenaf powders pretreated in ChFor, ChOAc, and ChPro by conventional heating at $110^{\circ} \mathrm{C}$ for $20 \mathrm{~min}$. In contrast, approximately $60 \%-90 \%$ of cellulose was hydrolyzed to glucose after microwave pretreatment in the same ILs at $110^{\circ} \mathrm{C}$ for $20 \mathrm{~min}$.
\end{abstract}

\title{
Keywords:
}

Ionic liquid; Microwave, Lignin; Cellulose; Cellulase; Bioconversion: 


\section{Introduction}

Lignocellulosic materials have gained attention as renewable sources of fermentable sugars for bioconversion into biofuel and platform chemicals, which are converted into value-added products [1]. These bioconversion processes generally consist of the following steps: i) pretreatment of lignocellulose to enhance the subsequent enzymatic saccharification of cellulose and hemicellulose, ii) enzymatic hydrolysis of cellulose and hemicellulose to fermentable sugars, and iii) microbial fermentation of the sugars to biofuel and platform chemicals. However, the crystalline and rigid structures of cellulose and lignin prevent hydrolytic enzymes from accessing the polysaccharides [2]. Therefore, the pretreatment of lignocellulosic biomass is an important unit operation for disrupting the hydrogen bonds in crystalline cellulose and the covalent cross-linkages in the lignin structure to ensure efficient enzymatic hydrolysis of lignocellulose.

Ionic liquids (ILs), which are generally defined as organic salts that melt below $100^{\circ} \mathrm{C}$, have recently received a great deal of attention because they are designer fluids, thermally stable, nonvolatile, and capable of dissolving polar and nonpolar organic, inorganic, and polymeric compounds under mild conditions [3,4]. After it was first reported that ILs are capable of dissolving cellulose [5], it has been demonstrated that cellulose re-precipitated after dissolution in ILs exhibits much higher enzymatic hydrolysis owing to its decreased crystallinity [6]. Moreover, after Fort et al. [7] demonstrated that ILs can dissolve biomass, several groups have applied this IL-assisted method of cellulose pretreatment to various lignocellulosic materials [8-10]. However, in these reports, the dissolution of lignocellulosic biomass in ILs was performed by conventional heating at approximately $110^{\circ} \mathrm{C}$. 
Recently, microwave technology has been recognized as a tool for the organic synthesis and processing of polymers [11,12]. Microwave heating is based on an internal heating process based on the direct absorption of energy by polar molecules, which differs from conventional heating that is based on heat transfer. With regard to applications of microwave heating to biomass dissolution in IL, Swatloski et al. [5] revealed that microwaves could increase the solubility of cellulose in IL. Moreover, previous studies have demonstrated that microwave irradiation enhances the dissolution of lignocellulosic materials in IL and subsequent fractionation of cellulose and lignin $[13,14]$. On the other hand, with regard to its application to IL-assisted biomass pretreatment for subsequent enzymatic hydrolysis, it has been recently demonstrated that microwave irradiation can facilitate the pretreatment of cellulose in IL $[15,16]$. However, to the best of our knowledge, there has been no report on microwave pretreatment of lignocellulosic materials in IL.

Therefore, in this study, we demonstrated that the pretreatment of lignocellulosic biomass could be enhanced by microwave heating, compared with conventional heating at $110^{\circ} \mathrm{C}$. In particular, we employed the "wholly bio-derived ILs" using cholinium cation combined with carboxylic acid-based anions ([Ch][CA] ILs; [17]), which have been previously demonstrated to be capable of pretreating lignocellulosic materials under conventional heating [18,19]. To validate the methodology of microwave pretreatment in cholinium ILs for the enzymatic hydrolysis of lignocellulose, enzymatic hydrolysis data were compared with data obtained using the existing method of thermal pretreatment in cholinium ILs.

\section{Materials and Methods}




\subsection{Lignocellulosic material and reagents}

Kenaf core fiber was supplied as a lignocellulosic material by Mitani Sangyo Co., Ltd. (Tokyo, Japan). The fiber was in the form of ground powder, with a size of approximately $1.0 \mathrm{~mm}$. As cholinium ILs, choline formate (ChFor), choline acetate (ChOAc), and choline propionate (ChPro) were prepared by a one-pot neutralization method as described previously [20], with minor modifications [18,19]. In brief, an equimolar amount of carboxylic acid was added dropwise to a choline hydroxide solution (45 wt\%) in methanol (Sigma-Aldrich, St. Louis, MO, USA) with cooling. The mixture was stirred at room temperature (RT) for $6 \mathrm{~h}$. Water and methanol were removed in vacuo using a rotary evaporator at $40^{\circ} \mathrm{C}$ for $1 \mathrm{~h}$ and then at $90^{\circ} \mathrm{C}$ for $2 \mathrm{~h}$. The resultant residue was dried under vacuum at RT for $16 \mathrm{~h}$. The water contents for the prepared ILs were determined to be below $0.5 \mathrm{wt} \%$ by Karl-Fischer titration (Mettler Toledo, DL31). Chemical structures of the prepared ILs were confirmed by ${ }^{1} \mathrm{H}-$ and

${ }^{13} \mathrm{C}-\mathrm{NMR}$. No peaks attributable to impurities were detected in the ${ }^{1} \mathrm{H}$ - and ${ }^{13} \mathrm{C}-\mathrm{NMR}$ spectra. Cellulase from Trichoderma viride [Meicelase, 6200 filter paper units (FPU) per gram] was kindly donated by Meiji Seika Pharma Co., Ltd. (Tokyo, Japan). All other chemicals were obtained from commercial sources and were of reagent grade.

\subsection{Microwave pretreatment for enzymatic hydrolysis}

Pretreatment for enzymatic hydrolysis was conducted by dissolving kenaf powder in ILs with continuous microwave irradiation, followed by regeneration and separation of the pretreated kenaf from IL. For the dissolution step, $0.25 \mathrm{~g}$ of kenaf powder was added to a test tube containing $5 \mathrm{~g}$ of IL. The kenaf/IL mixture in the test tube was irradiated with an output power of $100 \mathrm{~W}$ at a frequency of $2450 \pm 50 \mathrm{MHz}$ using a 
microwave reactor (green motif·Ic, IDX Co. Ltd., Sano, Japan) maintained at $110^{\circ} \mathrm{C}$ for 0-20 min. As a control pretreatment, the kenaf/IL mixture in the test tube was heated at $110^{\circ} \mathrm{C}$ in a dry oven with magnetic stirring (1200 rpm) for $0-20 \mathrm{~min}$. Another round of control pretreatments was performed at $90^{\circ} \mathrm{C}$ using $50 \mathrm{mM}$ phosphate buffer $(\mathrm{pH}=5.0)$ instead of ILs.

For recovering the dissolved kenaf from IL, the kenaf/IL mixture was diluted with $45 \mathrm{~mL}$ of deionized water in a $50-\mathrm{mL}$ tube, resulting in the precipitation of kenaf. After stirring, the $50-\mathrm{mL}$ tube was centrifuged $(8000 \times \mathrm{g})$ for $10 \mathrm{~min}$ at $25^{\circ} \mathrm{C}$, and the supernatant were removed. The washing procedure was repeated five times to remove IL. The recovered kenaf was then dried in an oven at $90^{\circ} \mathrm{C}$ for $24 \mathrm{~h}$, gravimetrically measured, and ground into powder using a homogenizer (Fastprep® FP100A, MP Biomedicals LLC., Solon, OH). The resultant kenaf powder was subjected to enzymatic hydrolysis and other analyses described below. The pretreatment and analyses were performed in duplicate.

\subsection{Compositional analysis}

Lignin and cellulose contents of kenaf powder were determined using TAPPI methods [21,22], with minor modifications. In brief, $0.1 \mathrm{~g}$ of kenaf powder was mixed with $2 \mathrm{~mL}$ of a $72 \%(\mathrm{v} / \mathrm{v}) \mathrm{H}_{2} \mathrm{SO}_{4}$ aqueous solution at RT for $2 \mathrm{~h}$. The mixture was then transferred to a $200-\mathrm{mL}$ Erlenmeyer flask, diluted with $75 \mathrm{~mL}$ of water, and autoclaved at $121^{\circ} \mathrm{C}$ for $15 \mathrm{~min}$. The dilute-acid hydrolysate was then filtered, following which the amount of acid-insoluble lignin was determined by gravimetrically measuring the residue on the filter after drying at $100^{\circ} \mathrm{C}$ for $12 \mathrm{~h}$. The amount of acid-soluble lignin was determined by UV absorbance of the filtrate at $205 \mathrm{~nm}$ and the absorption 
coefficient of $110 \mathrm{~L} \mathrm{~g}^{-1} \mathrm{~cm}^{-1}$ [21]. The sum of both acid-insoluble and acid-soluble lignin was regarded as lignin. The amount of glucose and xylose in the hydrolysate was determined by HPLC equipped with an RI detector (Shimadzu Co., Kyoto, Japan) using a CARBOSep CHO-682 column (Tokyo Chemical Industry Co., Ltd, Tokyo, Japan). The operating conditions were a temperature of $85^{\circ} \mathrm{C}$ with a water mobile phase and a flow rate of $0.4 \mathrm{~mL} / \mathrm{min}$. The amount of cellulose and hemicellulose was calculated from glucose and xylose contents multiplied by anhydro correction factors of 162/180 and $132 / 150$, respectively.

\subsection{Enzymatic hydrolysis}

Enzymatic hydrolysis of kenaf powder was conducted in $30-\mathrm{mL}$ vials at $50^{\circ} \mathrm{C}$ using a rotary shaker at $130 \mathrm{rpm}$. The reaction mixture consisted of $0.03 \mathrm{~g}$ of kenaf powder and $5 \mathrm{~mL}$ of cellulase solution $(62 \mathrm{FPU} / \mathrm{mL})$ in $50 \mathrm{mM}$ phosphate buffer $(\mathrm{pH}=$ 5.0) with $1 \%$ toluene to prevent contamination. Samples were collected after 0 and $48 \mathrm{~h}$ and then heated at $90^{\circ} \mathrm{C}$ for 5 min to inactivate the enzyme. After centrifugation of the heated sample at $21,500 \times \mathrm{g}$ for $1 \mathrm{~min}$, the glucose and xylose concentration of the supernatant was determined using the HPLC as described above. The cellulose saccharification was evaluated as the percentage of cellulose hydrolyzed into glucose to cellulose in kenaf recovered from the pretreatment. The hemicellulose saccharification was also evaluated as the percentage of hemicellulose hydrolyzed into xylose to hemicellulose in kenaf recovered from the pretreatment.

\section{Results and Discussion}

\subsection{Recovery and component of lignocellulosic biomass after microwave pretreatment}


in IL

As pretreatment for enzymatic hydrolysis, kenaf powder was dissolved in ILs with continuous microwave irradiation for 0-20 min, following which it was recovered as a precipitate by adding antisolvents (water). The percentage of recovered kenaf powder was approximately $80 \%$ of the untreated originals, regardless of the solvent (buffer or ILs), external energy (heat or microwave), and pretreatment time (Fig. 1). This indicates that a $10 \%-20 \%$ loss of kenaf powder is inevitable and intrinsic during the pretreatment and washing steps [23].

To examine the composition of kenaf powder pretreated by microwave irradiation in ILs, cellulose and lignin contents were determined. Untreated kenaf powder contained approximately 35\% cellulose, 30\% lignin, and 35\% hemicellulose (Fig. 2). The composition did not change significantly after pretreatment for up to $20 \mathrm{~min}$ regardless of whether the samples were pretreated by heating or microwave irradiation, when phosphate buffer was used instead of IL (Fig. 2A). On the other hand, slight decrease was observed in the percentage of cellulose and hemicellulose with the prolonged microwave pretreatment in the IL. This might be attributable to that a partially hydrolyzed (hemi)cellulose was lost during washing of the IL. However, it was suggested that microwave irradiation did not excessively degrade the specific macromolecules in kenaf powder under the examined conditions.

\subsection{Enzymatic hydrolysis of lignocellulosic biomass after microwave pretreatment in IL}

To examine the effects of microwave heating during pretreatment in ILs on subsequent enzymatic hydrolysis, kenaf powder pretreated for 0-20 min was subjected to enzymatic hydrolysis for $48 \mathrm{~h}$. The cellulose saccharification percentage reached 
saturation values within $48 \mathrm{~h}$, irrespective of the solvent (buffer or ILs) and pretreatment time (Fig. 3). Except for the cases involving phosphate buffer (Fig. 3A), the cellulose saccharification percentage increased with an increase in microwave pretreatment time (Fig. 3B-D). Thus, microwave irradiation enhances the efficacy of lignocellulose pretreatment in cholinium ILs.

A set of control experiments was also conducted using conventional heating. In the experiment where phosphate buffer was used instead of IL, the cellulose saccharification percentage was approximately $20 \%$ after pretreatment for up to $20 \mathrm{~min}$, regardless of whether the samples were pretreated by heating or microwave irradiation (Fig. 4A). The cellulose saccharification percentage remained at approximately $20 \%$ throughout the pretreatment time for up to $20 \mathrm{~min}$, even for kenaf powder thermally pretreated in ChFor, ChOAc, and ChPro (open keys in Fig. 4B-D). In contrast, for kenaf powder pretreated by microwave irradiation in these ILs, the cellulose saccharification percentage significantly increased with an increase in pretreatment time (closed keys in Fig. 4B-D). Specifically, almost $90 \%$ of cellulose was converted into glucose when kenaf powder was pretreated in ChOAc and ChPro for 20 min. Moreover, the microwave-assisted pretreatment also enhanced the hemicellulose saccharification percentage (Fig. 5). Here, the glucose yield and xylose yield on original biomass basis were also calculated considering the biomass recovery (Fig. 1) and the compositional change (Fig. 2) (see Fig S1 and S2, respectively), indicating that the microwave-assisted pretreatment enhanced the yields. Thus, compared with the conventional pretreatment method using cholinium IL and heating, pretreatment with a combination of cholinium IL and microwave irradiation can significantly enhance the enzymatic hydrolysis of kenaf powder. 


\subsection{Comparison between microwave and conventional heating for biomass pretreatment in ILs}

We previously demonstrated that the cellulose saccharification percentage in kenaf powder reached almost $90 \%$ when the biomass was pretreated using [Ch][CA] ILs under conventional heating at $110^{\circ} \mathrm{C}$ for $16 \mathrm{~h}$ [18]. However, the present study revealed that microwave pretreatment in ILs could not only achieve the same levels of cellulose saccharification but also drastically reduce the pretreatment time to $20 \mathrm{~min}$. This enhanced efficiency by microwave pretreatment in ILs may be attributable to the following: 1) Microwave heating is characterized by an internal heating process owing to the direct absorption of energy by polar molecules. ILs can efficiently absorb microwave radiation because they only consist of anions and cations [24,25]. Thus, ILs with high polarity could be easily heated by microwave irradiation, and the resultant internal heating may be responsible for the more effective breakdown of the H-bond network between cellulose microfibrils. 2) Microwave enhanced the diffusion of IL molecules and collision frequency between anions and cations of IL and the wood macromolecules. This may facilitate the inter- and intramolecular hydrogen bonding interactions between cellulose microfibrils and anions of IL, resulting in more rapid pretreatment. There was no positive effect of microwave pretreatment in phosphate buffer on the cellulose saccharification percentage, indicating that microwave irradiation itself had no sufficient pretreatment effect to enhance enzymatic hydrolysis under the examined conditions (closed keys in Fig. 4A). Therefore, the combination of microwave irradiation and ILs synergistically enhanced the cellulose saccharification percentage of kenaf powder. 
With regard to energy saving, Wang et al. [14] compared the energy requirement for microwave heating and conventional heating during biomass treatment in ILs. They approximately estimated that the energy required in microwave treatment in ILs was much less than that required in conventional heating. The energy required in microwave pretreatment was also calculated under the conditions employed in the present study. By multiplying the power $(100 \mathrm{~W})$ with pretreatment time $(20 \mathrm{~min})$, the energy required for pretreatment with microwave heating is determined to be $1.2 \times 10^{5} \mathrm{~J}$. On the other hand, the energy required for pretreatment with conventional heating at $110^{\circ} \mathrm{C}$ for $16 \mathrm{~h}$ is determined to be $1.1 \times 10^{7} \mathrm{~J}$ [14]. Thus, it was suggested that the combination of microwave irradiation and ILs could save energy for pretreatment as well as enhance pretreatment efficiency for cellulose saccharification.

For large-scale application of ILs to the biomass pretreatment process, development of efficient and energy-saving IL recovery methods is also important. Evaporation or ion-exclusion chromatography has been generally used to recycle ILs; however, these methods are costly and complex for large-scale application. Therefore, further research on IL recovery methods is necessary for realization of the IL-based biomass pretreatment process.

\section{Conclusions}

Microwave pretreatment in ILs was more effective than conventional thermal pretreatment in ILs for enzymatic hydrolysis of kenaf powders. Approximately $40 \%-70 \%$ improvement in the cellulose saccharification percentage was obtained with microwave pretreatment at $110^{\circ} \mathrm{C}$ in $\mathrm{ChFor}$, ChOAc, and ChPro for 20 min compared with that obtained with thermal pretreatment at $110^{\circ} \mathrm{C}$ in these ILs. 


\section{Acknowledgements}

This study was supported in part by an Adoptable and Seamless Technology Transfer Program through Target-driven R\&D (A-STEP) (No. A2121248E to K.N.) of the Japan Science and Technology Agency (JST) of Japan. This study was supported in part by an Advanced Low Carbon Technology Research and Development Program (ALCA) (No. 2100040 to K.T.) of the Japan Science and Technology Agency (JST) of Japan.

\section{References}

[1] M.G. Adsul, M.S. Singhvi, S.A. Gaikaiwari, D.V. Gokhale, Development of biocatalysts for production of commodity chemicals from lignocellulosic biomass, Bioresour. Technol. 102 (2011) 4304-4312.

[2] P. Alvira, E. Tomás-Pejó, M. Ballesteros, M.J. Negro, Pretreatment technologies for an efficient bioethanol production process based on enzymatic hydrolysis: A review, Bioresour. Technol. 101 (2010) 4851-4861.

[3] H. Olivier-Bourbigou, L. Magna, D. Morvan, Ionic liquids and catalysis: Recent progress from knowledge to applications, Appl. Catal. A 373 (2010) 1-56.

[4] N. Sun, H. Rodríguez, M. Rahman, R.D. Rogers, Where are ionic liquid strategies most suited in the pursuit of chemicals and energy from lignocellulosic biomass?, Chem. Commun. 47 (2010) 1405-1421.

[5] R.P. Swatloski, S.K. Spear, J.D. Holbrey, R.D. Rogers, Dissolution of cellulose with ionic liquids, J. Am. Chem. Soc. 124 (2002) 4974-4975.

[6] A.P. Dadi, S. Varanasi, C.A. Schall, Enhancement of cellulose saccharification 
kinetics using an ionic liquid pretreatment step, Biotechnol. Bioeng. 95 (2006) 904-910.

[7] D.A. Fort, R.C. Remsing, R.P. Swatloski, P. Moyna, G. Moyna, R.D. Rogers, Can ionic liquids dissolve wood? Processing and analysis of lignocellulosic materials with 1-n-butyl-3-methylimidazolium chloride, Green Chem. 9 (2007) 63-69.

[8] I. Kilpeläinen, H. Xie, A. King, M. Granstrom, S. Heikkinen, D.S. Argyropoulos, Dissolution of wood in ionic liquids, J. Agric. Food Chem. 55 (2007) 9142-9148.

[9] S.H. Lee, T.V. Doherty, R.J. Linhardt, J.S. Dordick, Ionic liquid-mediated selective extraction of lignin from wood leading to enhanced enzymatic cellulose hydrolysis, Biotechnol. Bioeng. 102 (2009) 1368-1376.

[10] Q. Li, Y.-C. He, M. Xian, G. Jun, X. Xu, J.-M. Yang, L.Z. Li, Improving enzymatic hydrolysis of wheat straw using ionic liquid 1-ethyl-3-methyl imidazolium diethyl phosphate pretreatment, Bioresour. Technol. 100 (2009) $3570-3575$.

[11] P. Lidström, J. Tierney, B. Wathey, J. Westman, Microwave assisted organic synthesis — a review, Tetrahedron. 57 (2001) 9255-9283.

[12] W.C. Conner, G.A. Tompsett, How could and do microwaves influence chemistry at interfaces?, J. Phys. Chem. B. 112 (2008) 2110-2118.

[13] X. Wang, H. Li, Y. Cao, Q. Tang, Cellulose extraction from wood chip in an ionic liquid 1-allyl-3-methylimidazolium chloride (AmimCl),, Bioresource Technol. 102 (2011) 7959-79565.

[14] H. Wang, M.L. Maxim, G. Gurau, R.D. Rogers, Microwave-assisted dissolution and delignification of wood in 1-ethyl-3-methylimidazolium acetate., Bioresource Technol. 136 (2013) 739-742. 
[15] L. Li, S.-T. Yu, F.-S. Liu, C.-X. Xie, C.-Z. Xu, Efficient enzymatic in situ saccharification of cellulose in aquaeous-ionic liquid media by microwave pretreatment, BioResource. 6 (2011) 4494-4504.

[16] S.H. Ha, N.L. Mai, G. An, Y.-M. Koo, Microwave-assisted pretreatment of cellulose in ionic liquid for accelerated enzymatic hydrolysis, Bioresource Technol. 102 (2011) 1214-1219.

[17] S. Hu, T. Jiang, Z. Zhang, A. Zhu, B. Han, J. Song, Y. Xie, W. Li, Functional ionic liquid from biorenewable materials: synthesis and application as a catalyst in direct aldol reactions, Tetrahedron Lett. 48 (2007) 5613-5617.

[18] K. Ninomiya, T. Yamauchi, M. Kobayashi, O. Chiaki, K. Takahashi, N. Shimizu, Cholinium carboxylate ionic liquids for pretreatment of lignocellulosic materials to enhance subsequent enzymatic saccharification, Biochem. Eng. J. 71 (2013) $25-29$.

[19] K. Ninomiya, H. Soda, C. Ogino, K. Takahashi, N. Shimizu, Effect of ionic liquid weight ratio on pretreatment of bamboo powder prior to enzymatic saccharification, Bioresour. Technol., 128, (2013) 188-192.

[20] Y. Yu, X. Lu, Q. Zhou, K. Dong, H. Yao, S. Zhang, Biodegradable naphthenic acid ionic liquids: synthesis, characterization, and quantitative structure-biodegradation relationship, Chem. Eur. J. 14 (2008) 11174-11782.

[21] TAPPI useful method UM-250, 1991. Acid-soluble lignin in wood and pulp.

[22] TAPPI method T $222 \mathrm{~cm}-02,2002$. Acid-insoluble lignin in wood and pulp.

[23] N. Sun, M. Rahman, Y. Qin, M.L. Maxim, H. Rodríguez, R.D. Rogers, Complete dissolution and partial delignification of wood in the ionic liquid 1-ethyl-3-methylimidazolium acetate, Green Chem. 11 (2009) 646-655. 
[24] J. Hoffmann, M. Nüchter, B. Ondruschka, P. Wasserscheid, Ionic liquids and their heating behaviour during microwave irradiation - a state of the art report and challenge to assessment, Green Chem. 5 (2003) 296-299

[25] L. Feng, Z.L. Chen, Research progress on dissolution and functional modification of cellulose in ionic liquids, J. Mol. Liq. 142 (2008) 1-5. 


\section{Figure captions}

Fig. 1 Percentages of kenaf powder recovered after pretreatment in ILs for prescribed times. Open circles: thermal pretreatment in IL; closed circles: microwave pretreatment in IL. (A) Phosphate buffer, (B) ChFor, (C) ChOAc, and (D) ChPro.

Fig. 2 Content of cellulose (bottom of stacked bars), hemicellulose (middle of stacked bars), lignin, and others (top of stacked bars) in kenaf powder pretreated in ILs for prescribed times. Open bars: thermal pretreatment in IL; closed bars: microwave pretreatment in IL. (A) Phosphate buffer, (B) ChFor, (C) ChOAc, and (D) ChPro.

Fig. 3 Time courses of cellulose saccharification percentage during enzymatic hydrolysis of kenaf powder pretreated in IL with microwave irradiation. Circles: pretreated for $0 \mathrm{~min}$; triangles: pretreated for $5 \mathrm{~min}$; squares: pretreated for 10 min; diamonds: pretreated for $15 \mathrm{~min}$; inverse triangles: pretreated for $20 \mathrm{~min}$. (A) Phosphate buffer, (B) ChFor, (C) ChOAc, and (D) ChPro.

Fig. 4 Cellulose saccharification percentages of kenaf powder pretreated in IL for prescribed times. The percentages were obtained $48 \mathrm{~h}$ after enzymatic hydrolysis reaction. Open circles: thermal pretreatment in IL; closed circles: microwave pretreatment in IL. (A) Phosphate buffer, (B) ChFor, (C) ChOAc, and (D) ChPro. 
Fig. 5 Hemiellulose saccharification percentages of kenaf powder pretreated in IL for prescribed times. The percentages were obtained $48 \mathrm{~h}$ after enzymatic hydrolysis reaction. Open circles: thermal pretreatment in IL; closed circles: microwave pretreatment in IL. (A) Phosphate buffer, (B) ChFor, (C) ChOAc, and (D) ChPro. 


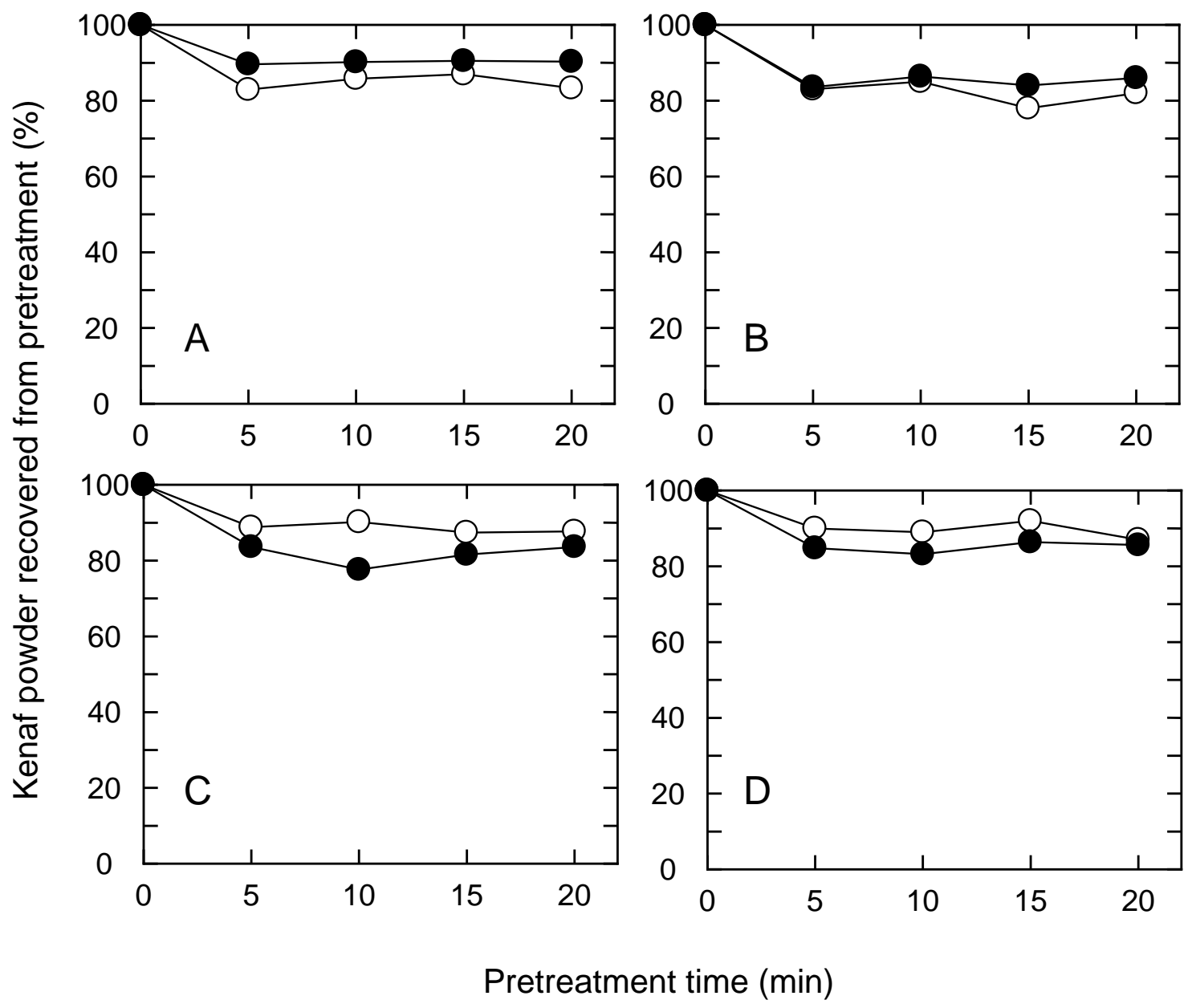

Fig. 1 Percentages of kenaf powder recovered after pretreatment in ILs for prescribed times. Open circles: thermal pretreatment in IL; closed circles: microwave pretreatment in IL. (A) Phosphate buffer, (B) ChFor, (C) ChOAc, and (D) ChPro. 

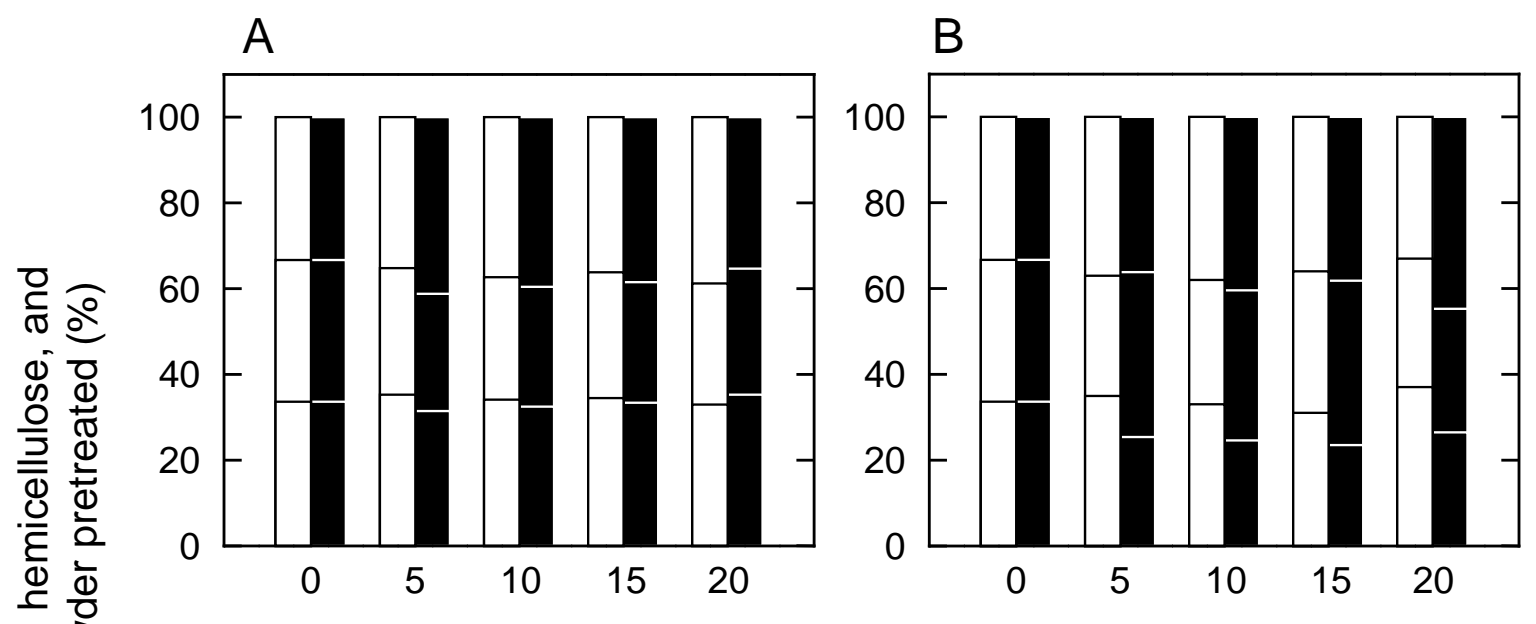

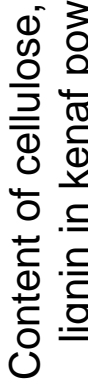
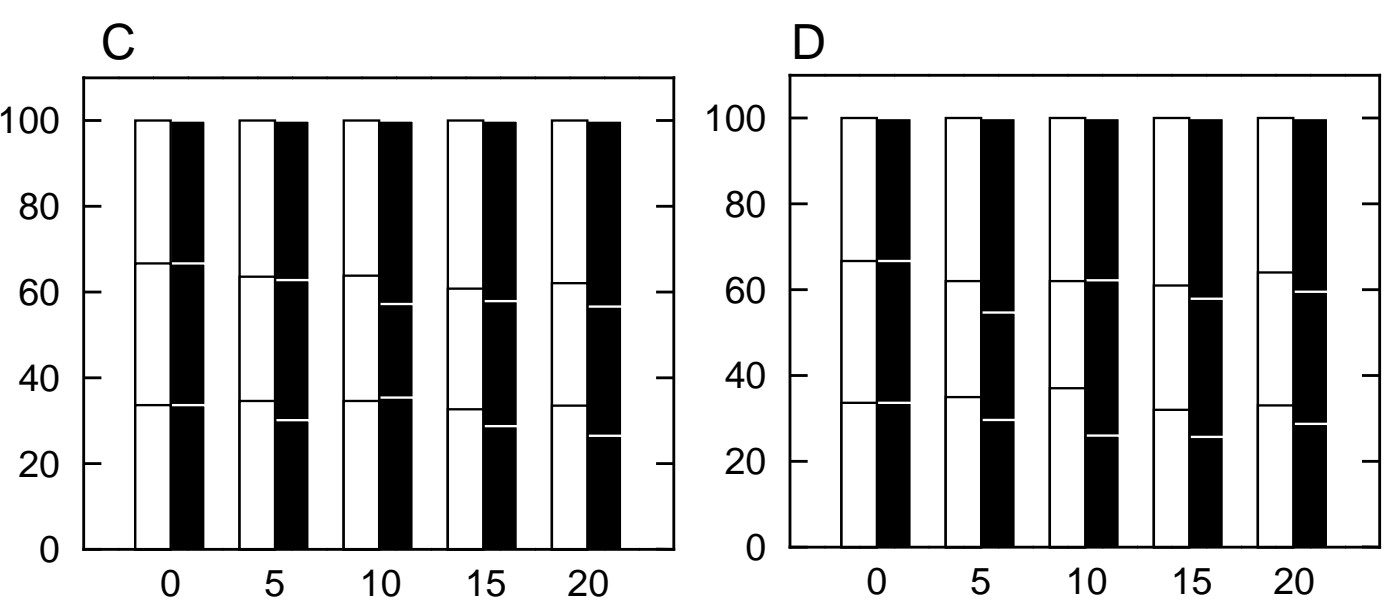

Pretreatment time (min)

Fig. 2 Content of cellulose (bottom of stacked bars), hemicellulose (middle of stacked bars), lignin, and others (top of stacked bars) in kenaf powder pretreated in ILs for prescribed times. Open bars: thermal pretreatment in IL; closed bars: microwave pretreatment in IL. (A) Phosphate buffer, (B) ChFor, (C) ChOAc, and (D) ChPro. 


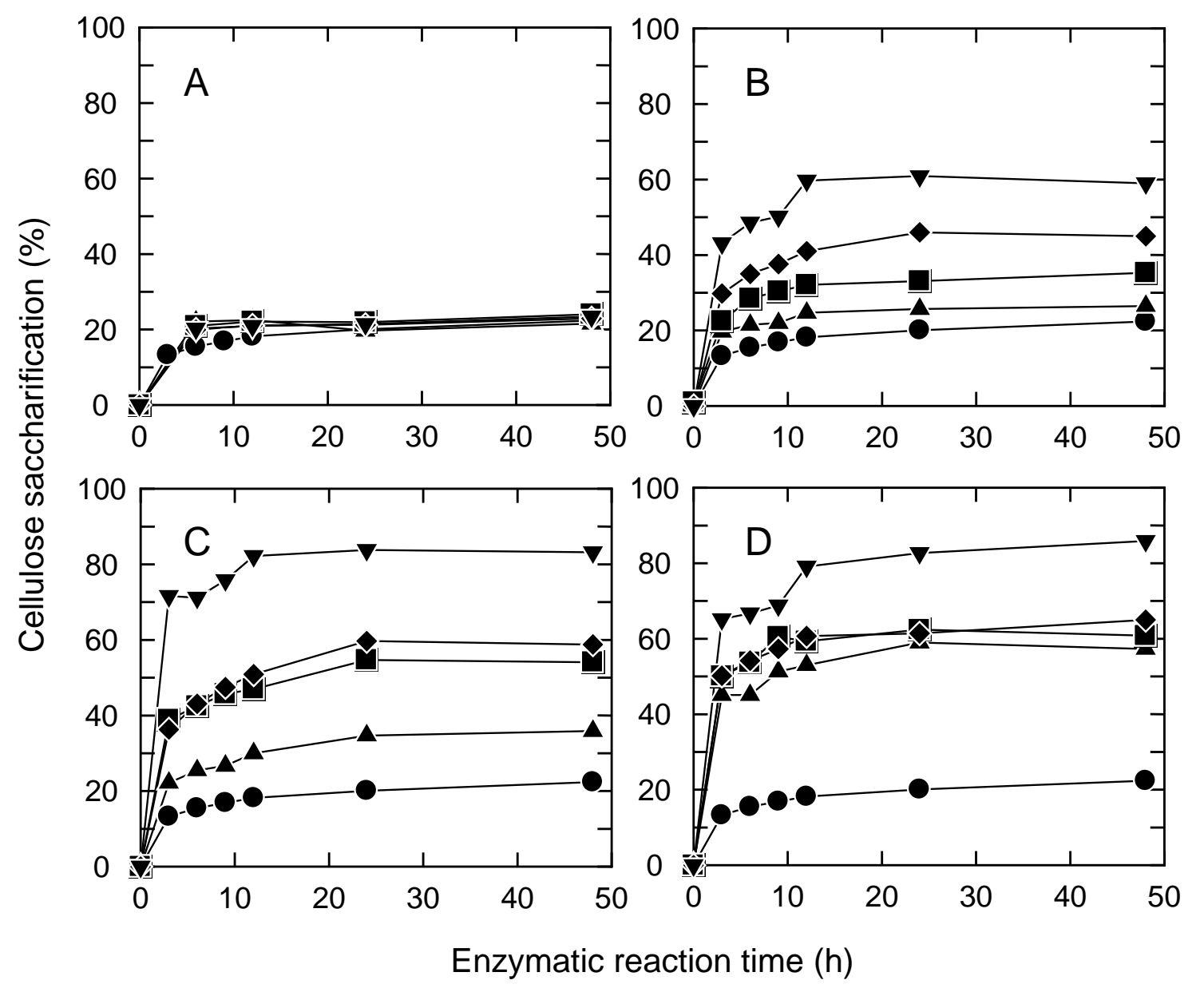

Fig. 3 Time courses of cellulose saccharification percentage during enzymatic hydrolysis of kenaf powder pretreated in IL with microwave irradiation. Circles: pretreated for $0 \mathrm{~min}$; triangles: pretreated for $5 \mathrm{~min}$; squares: pretreated for $10 \mathrm{~min}$; diamonds: pretreated for $15 \mathrm{~min}$; inverse triangles: pretreated for $20 \mathrm{~min}$. (A) Phosphate buffer, (B) ChFor, (C) ChOAc, and (D) ChPro. 


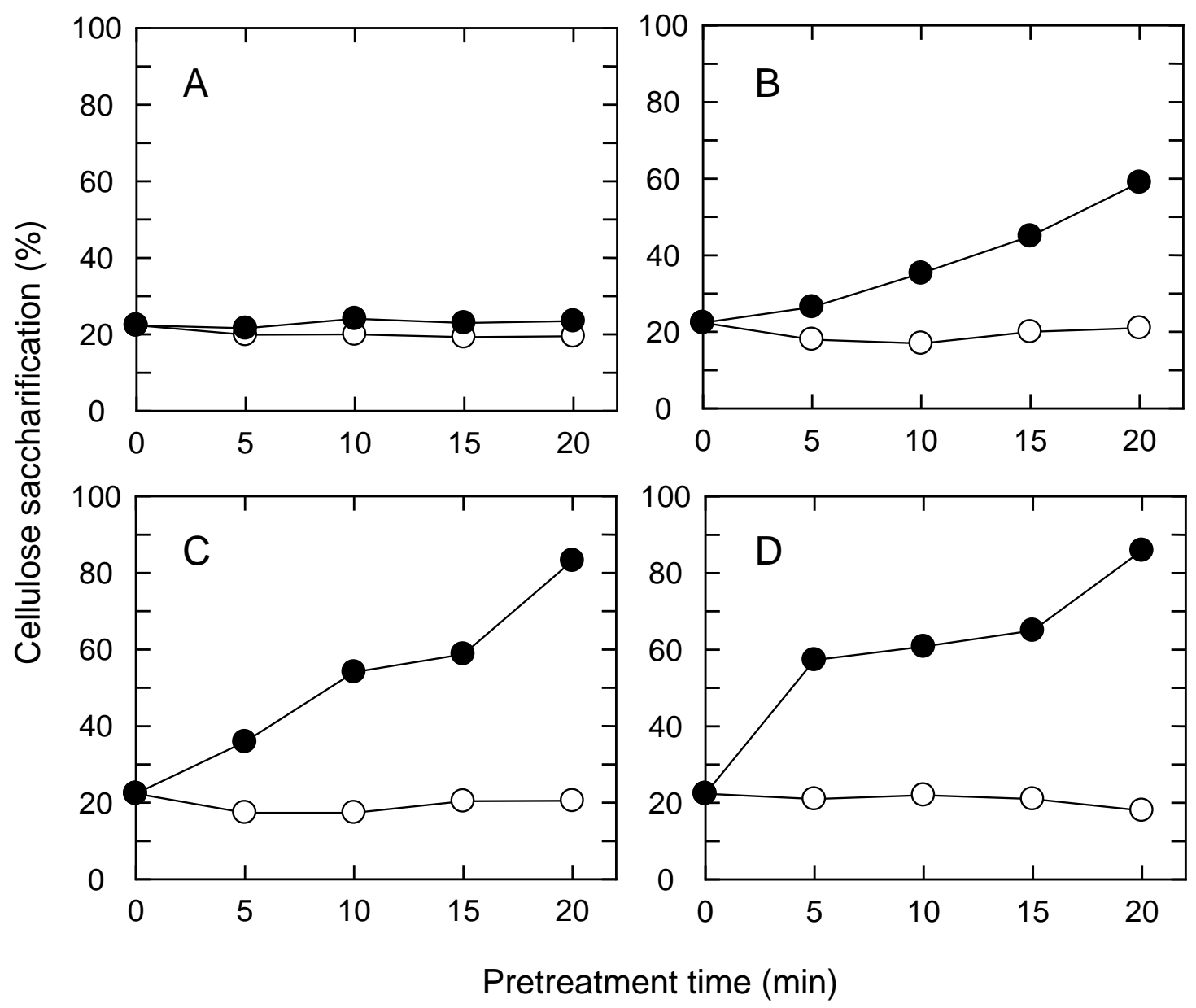

Fig. 4 Cellulose saccharification percentages of kenaf powder pretreated in IL for prescribed times. The percentages were obtained $48 \mathrm{~h}$ after enzymatic hydrolysis reaction. Open circles: thermal pretreatment in $\mathrm{IL}$; closed circles: microwave pretreatment in IL. (A) Phosphate buffer, (B) ChFor, (C) ChOAc, and (D) ChPro. 


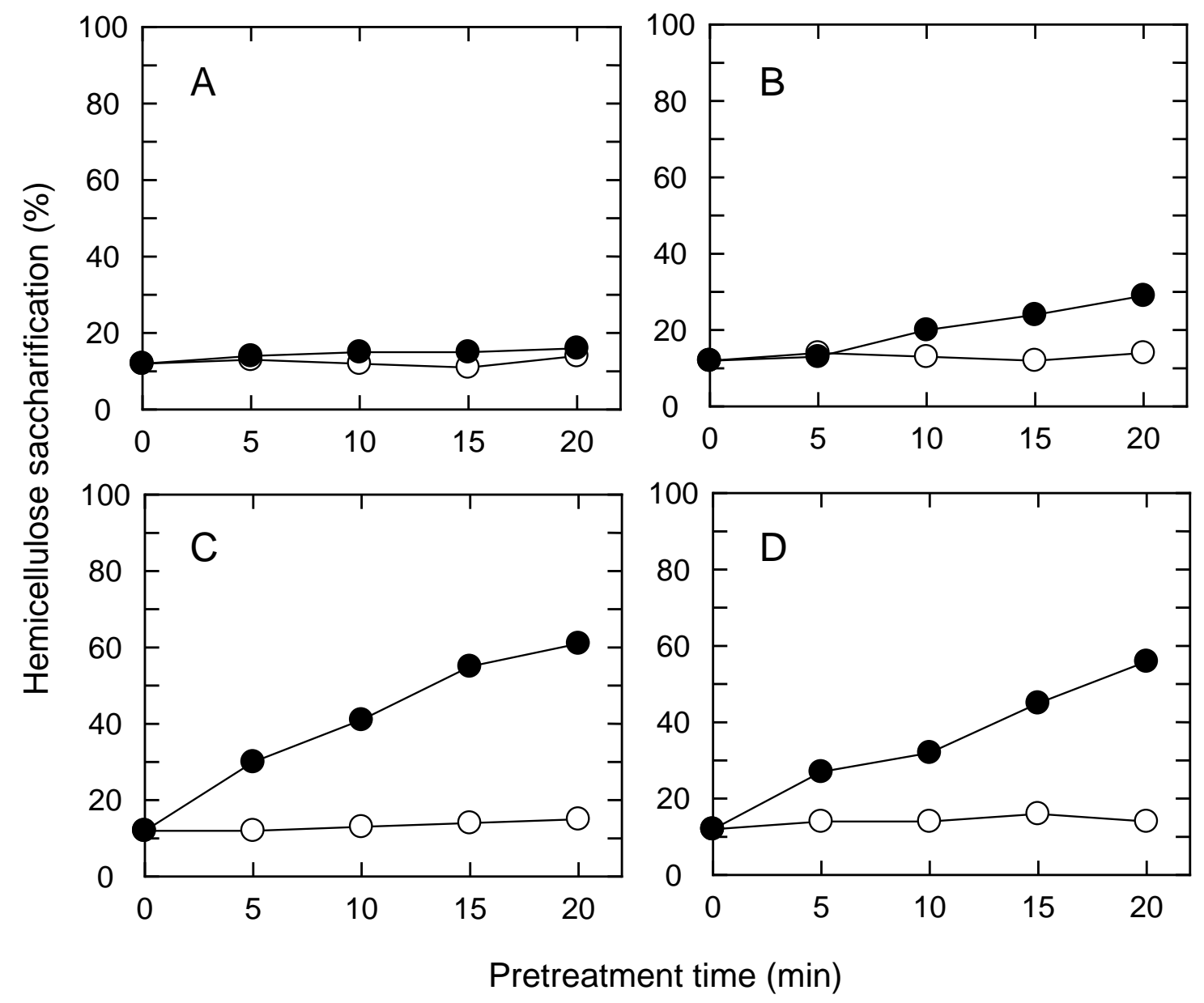

Fig. 5 Hemicellulose saccharification percentages of kenaf powder pretreated in IL for prescribed times. The percentages were obtained $48 \mathrm{~h}$ after enzymatic hydrolysis reaction. Open circles: thermal pretreatment in IL; closed circles: microwave pretreatment in IL. (A) Phosphate buffer, (B) ChFor, (C) ChOAc, and (D) ChPro. 